\title{
Use of endoscope as adjunct to microscope in cholesteatoma surgery-A non-randomized, non-controlled study
}

\author{
Bhavika Verma, Yogesh Dabholkar*, Haritosh Velankar, and Akanksha A Saberwal \\ Department of ENT, D.Y.Patil University School of Medicine, Nerul, Navi Mumbai, India
}

\begin{abstract}
Importance; The use of endoscope for the ear has opened up new approaches to treat cholesteatoma surgically and reduce residuals. The endoscope can be used as an adjunct to the microscope or by itself.

Aims and objectives:

1. To evaluate the use of endoscopy in identifying the cholesteatoma remnant at the time of primary surgery with the operating microscope.

2. To evaluate the effectiveness of endoscopy in assessing limited disease during cholesteatoma surgery.

Design: A hospital- based, interventional, non-randomized, non-comparative and prospective study was done in 116 ears with acquired cholesteatoma. At the time of the surgery, the endoscope was used to study and identify the extent of the disease. After completion of the surgery by the standard inside-out technique using microscope and drill, the endoscope was used to identify the cholesteatoma remnants if any.
\end{abstract}

Setting: This study was done in a tertiary care hospital.

Participants: 116 patients with acquired cholesteatoma that were operated on.

Main outcome measure: The proportion of residual disease identified with the help of endoscope at the end of standard surgery using microscope and drill. Secondary outcome measure was the proportion of cases in which endoscope was useful in decision making intra-operatively.

Results: Out of 116 patients operated with the microscope, 13 had a cholesteatoma remnant at the end of surgery which was missed by the microscope but identified with the oto-endoscope. The sinus tympani was the commonest site of cholesteatoma remnant. Also in 7 cases, the endoscope helped in limiting the dissection by better identification of the extent of disease as compared to microscope during surgery.

Conclusion and relevance: Endoscope is a useful adjunct to the operating microscope in cholesteatoma surgeries. It is useful in not only identifying residual disease but also in decision making by identifying the extent of the disease intra-operatively.

\section{Introduction}

Cholesteatoma is a cyst-like, expansile lesion of the temporal bone lined by stratified squamous epithelium with a tendency to erode surrounding bone, ossicles, facial canal, inner ear or intracranial cavity causing hearing loss, facial paralysis, vertigo or intracranial complications.

The widely accepted treatment of cholesteatoma is surgical excision by canal wall down (CWD) mastoidectomy. This technique provides good surgical exposure for cholesteatoma removal and yields significantly lower rate of residual cholesteatoma than canal wall up procedure, although canal wall up procedure has lower morbidity and better functional outcome $[1,2]$.

Inadvertent incomplete removal leading to residual disease may happen even after meticulous removal of the matrix under operating microscope, due to presence of cholesteatoma in hidden areas, not readily visualized by straight-line vision of the microscope.

These limitations can be overcome with the complementary help of the endoscope, which allows 'looking around corners' [3,4].
Thomassin et al. [5] reported a significant reduction in residual cholesteatoma rates by using endoscopes as adjunct to microscope in cholesteatoma surgery. He attributed it to better visualization of blind spots with endoscopes. Bottrill and Poe [6], in 1995 demonstrated a reduced morbidity and increased accuracy of surgical resection with endoscopes as adjunct to microscope. Tarabichi [7], who has the largest series of cholesteatoma surgeries with the endoscope, has even described exclusively endoscopic management of cholesteatoma.

However, the use of endoscope, even as adjunct to microscope, has not gained wide acceptance for cholesteatoma surgery. The use of endoscope to assess the extent of the disease intra-operatively and

Correspondence to: Yogesh Dabholkar, Department of ENT, D.Y.Patil University School of Medicine, Nerul, Navi Mumbai, India, Tel: +91- 9967902777; Email: ygsh@yahoo.com

Key words: Otoendoscopy, residual cholesteatoma, mastoidectomy

Received: December 03, 2017; Accepted: December 22, 2017; Published: December 26, 2017 
its role in decision making in cholesteatoma surgery is yet to be fully explored.

The present study was undertaken to investigate the use of endoscopes as adjunct to microscope in identifying residual disease in hidden areas and its role in decision making in cholesteatoma surgery.

\section{Materials and methods}

Prior ethical approval was taken from the institutional ethical committee.

This study was conducted from December 2014 to December 2016. Pregnant women and children were excluded from the study. Also, cases with intracranial complications were excluded from the study. One hundred and sixteen cases with active squamosal disease as identified by ear microscopy and endoscopy were included in the study.

This interventional, non-randomized, non-comparative and prospective study was done in a university hospital.

A customized proforma was used for collection of data for every patient. A detailed history, examination and relevant investigations including ear microscopy and endoscopy were recorded preoperatively for all patients.

All cases were operated under general anesthesia by either of four surgeons with varied surgical experience ranging from five to twenty years.

A standard inside-out (anterior-posterior) technique was used to excise the cholesteatoma in all patients with a Carl Zeiss Movena microscope and Medtronic micro drill with standard microsurgical instruments under continuous suction irrigation. In cases in which a limited disease was encountered, the endoscope was also used as an adjunct to the microscope to visualize the end of the sac. The extent of bone-dissection performed was governed by intra-operative evaluation of disease extension and ranged from a limited atticotomy to canal wall down mastoidectomy. The percentage of cases in which the endoscope was useful to reach a decision for limited dissection was calculated.

Once the microscopic resection was completed and there was no visible cholesteatoma under the microscope, 0 degree and 45 degree, $4 \mathrm{~mm}$ Hopkins rod telescopes (Karl Storz GmbH \& Co., Tuttlingen, Germany) along with a Tricam SL-II video camera (Karl Storz GmbH $\&$ Co.) were used to assess the middle ear for presence of any remaining disease with special attention to 'hidden areas' like sinus tympani and facial recess. Any such remaining disease was removed with help of endoscope and microscope. The percentage of cases identified by endoscope to have remnant cholesteatoma even after completion of surgery with microscope was calculated.

Tympanic reconstruction was done with temporalis fascia or split thickness cartilage. Cartilage was used to reconstruct the lateral attic wall defect in cases requiring an atticotomy. The ossicular reconstruction if required was done in same sitting (single-stage). Standard postoperative management was carried out for all patients. Patients were followed up at three and six months post-surgery for any residual or recurrence of cholesteatoma.

\section{Results}

The male female ratio was $3: 2$. The age of the patients was ranging from 18 to 55 years (Tables 1 and 2).

After completion of disease clearance with microscope, the endoscope confirmed absence of any residual epithelium in 103 cases
Table 1. Site-wise and extent-wise surgical resection of cholesteatoma.

\begin{tabular}{|c|c|c|c|}
\hline $\begin{array}{c}\text { Location of } \\
\text { Cholesteatoma }\end{array}$ & Number of Cases & $\begin{array}{c}\text { Transcanal } \\
\text { excision (including } \\
\text { atticotomy) }\end{array}$ & $\begin{array}{c}\text { CWD } \\
\text { Mastoidectomy }\end{array}$ \\
\hline Attic Cholesteatoma & 53 & 22 & 31 \\
\hline $\begin{array}{c}\text { Postero-superior } \\
\text { cholesteatoma }\end{array}$ & 44 & 17 & 27 \\
\hline $\begin{array}{c}\text { Postero-inferior } \\
\text { cholesteatoma }\end{array}$ & 16 & 13 & 03 \\
\hline $\begin{array}{c}\text { Anterior TM } \\
\text { cholesteatoma }\end{array}$ & 03 & 3 & 0 \\
\hline
\end{tabular}

Table 2. Site-wise remnant cholesteatoma in hidden areas identified with endoscope at the end of surgery with microscope.

\begin{tabular}{|c|c|}
\hline Sites of cholesteatoma remnant & Number (Percentage) of Cases \\
\hline Sinus Tympani & $7(6.03)$ \\
\hline Anterior Epitympanic Recess & $2(1.72)$ \\
\hline Mesotympanum & $0(0)$ \\
\hline Hypotympanum & $0(0)$ \\
\hline Facial Recess & $4(3.44)$ \\
\hline Attic & $0(0)$ \\
\hline Around the ossicles & $2(1.72)$ \\
\hline E.T. Opening & $0(0)$ \\
\hline Area of Cog & $0(0)$ \\
\hline Mastoid Tip & $0(0)$ \\
\hline
\end{tabular}

(88.79\%). However, in 13 cases $(11.2 \%)$, the endoscope could identify residual epithelium in hidden areas which was not visualized by microscope. In two cases there were cholesteatoma remnant at two sites.

A canal wall down mastoidectomy was required in 61 cases (52.58\%), whereas in 55(47.41\%) cases the disease was limited either to the middle ear and/or epitympanum and could be completely excised with a transcanal technique.

Out of the 55 cases with a limited extent, in 48 cases the end of the sac could be identified with microscope itself and in 7 cases where there was a doubt about the extent, adjunctive endoscopy was useful in confirming the end of the disease. Further dissection and a canal wall down mastoidectomy could thus be averted in these 7 cases. After excising the sac the endoscope also confirmed absence of any residual disease in all these cases (Table 3 ).

\section{Discussion}

Cholesteatoma surgery primarily aims to eradicate the disease process and provide the patient with a safe and dry ear. The main problem in cholesteatoma removal is recidivism either residual disease or recurrence. The residual disease is often due to inadvertent, incomplete resection as a result of poor exposure of hidden areas such as the sinus tympani and facial recess. The anatomy of middle ear is extremely complex making it occasionally difficult for the surgeon to visualize entire middle ear space with direct line vision of the microscope. Despite the illumination and magnification offered by the operating microscope, it has distinct limitations of visualization of hidden areas and inability to look around corners. This often necessitates the use of inefficient, blind, blunt probing to clear disease in hidden areas.

The endoscopic approach has led to better surgical treatment options for middle ear pathologies by affording round-the-corner views of hidden areas such as sinus tympani, facial recess, anterior epitympanic spaces, attic, hypotympanum and protympanum [8-10].

Shelton and Sheehy in 1990 [11] reported residual cholesteatoma in one third of their cases and attributed it to poor control over areas 
Table 3. Identification of end of sac.

\begin{tabular}{|c|c|}
\hline Identification of end of sac & Number (percentage) of cases \\
\hline Microscope alone & $48(87.27)$ \\
\hline Microscope with endoscope & $7(12.72)$ \\
\hline
\end{tabular}

difficult to reach with the microscope. Dodson et al. [12] in 1988 observed that residual disease may reach up to $42 \%$ in canal wall up technique and $12 \%$ in canal wall down technique. Biswas and Mandal [13] reported intraoperative residual cholesteatoma rates of $13 \%$ in CWU mastoidectomy operation and $11.8 \%$ in CWD mastoidectomy operation when using microscope only and no residual cholesteatoma when using endoscope along with microscope.

In our study after completion of mastoidectomy using microscope, epithelial remnants were detected by the endoscope in hidden areas in $11.2 \%$ of cases. Sinus tympani was the commonest area where cholesteatoma matrix missed by the microscope was identified using endoscope $(6.03 \%$ of all cases), whereas the facial recess was second most common site (3.44\%). In all these cases the disease remnants once identified by endoscope were removed successfully with a combination of microscope and endoscope.

Thomasin et al. [5] in 2007 reported sinus tympani as the main site of recurrence. According to them sinus tympani remains the most inaccessible site in the middle ear and can be challenging to address when diseased.

Magnan et al. [14] and Pratt [15] who also found a higher incidence of recidivism in sinus tympani area. This may be due to poor visualization of sinus tympani by the microscope leading to blind attempts at Cholesteatoma removal by blunt probing instead of the regular technique of elevating matrix under vision.

Sinus tympani and facial recess could not be visualized completely in all our cases by microscope alone, even after a canal wall down. However, the use of endoscope enabled visualization of hidden areas in these cases allowing a complete resection. Endoscopes provide better control over pathology in areas which are difficult to visualize [16]. The use of endoscopes has been likewise demonstrated to achieve a significantly higher degree of control over middle ear disease and dramatically reduce the incidence of cholesteatoma recurrence particularly in hidden recesses such as sinus tympani [17].

Another important anatomical area that remains difficult to explore using the operative microscope is the anterior epitympanic space (AES) $[18,19]$. In our study, AES is a less commonly affected hidden space of the middle-ear cleft at about $1.72 \%$ ( 2 out of 116 patients with epithelial remnants).

However, Migirov et al. [20], have reported a relatively higher rate of cholesteatoma involvement in the AES in their study.

In our study, 61 cases required a canal wall down mastoidectomy as the disease on being followed by inside out technique was found to extend to the mastoid antrum. In 55 cases the disease was limited to middle ear and/or attic and could be managed by a transcanal technique (either a tympanoplasty or a tympanoplasty with atticotomy and lateral attic wall reconstruction with cartilage). Out of these 55 cases, the endoscope scored over the microscope by successfully identifying the end of the sac in 7 cases and thereby avoiding a canal wall down procedure. We believe that the endoscope can look around corners and identify the end of the sac better than microscope in inside-out technique, and thus prove useful in avoiding a large cavity post-operatively.
All patients in our series were followed up 3 months, 6 months and 1 year after surgery. The patients underwent microscopic as well as otoendoscopic examination at follow up. At the end point of our study, 1year post-surgery, none of our patients had any sign of recurrent or residual cholesteatoma. It is needless to say that long-term follow-up is important when discussing the recurrence rate of cholesteatoma. The rate of recurrence increases with follow-up time. In our study, though follow-up is relatively short, further observations are planned to assess long-term results.

There was neither morbidity nor complications secondary to the use of endoscopes in current study. However, the risk of damage to ossicular chain should not be underestimated. Thomassin et al. [5] reported two cases of total sensorineural hearing loss as a result of trauma to the intact stapes.

Endoscopic ear surgery has some limitations [18]:

1. Results depend on the experience of the surgeon with endoscopic approach as there is a steep learning curve.

2. Trauma from the tip of endoscope to facial nerve, ossicular chain, and low-lying tegmen especially in inexperienced hands.

3. In endoscopic ear surgery, one hand is occupied with endoscope and the other performs the manipulations. Hence, assistance is often required with the operating microscope when there is a need for two-hand manipulations for clearing the cholesteatoma or for ossicular reconstruction.

4. Loss of depth perception and a dissociation of visual and motor axis in camera assisted endoscopic surgery which is later compensated with experience.

5. Fogging and smearing of the tip of the endoscope and the need for frequent cleaning and application of defogging agents.

Owing to these limitations, in our study, the endoscope was used only as an adjunct to the microscope for performing cholesteatoma surgeries.

Many authors, such as Badr-El-Dine [21], Thomassin et al. [5], Magnan et al. [14], and Pratt [15] preferred the use of adjunctive endoscopy to traditional mastoidectomy surgeries with microscope alone, whereas McKennan [22] and Youssef and Poe [23] restricted the use of the endoscope to only the second-look approach, introducing the optic through the mastoid cavity and exploring hidden recesses.

Although endoscopy cannot be viewed as a replacement for microscope for mastoid surgery, it can be used as an additional tool, owing to its ability to look around corners and thus detect residual disease in hidden areas and identify the precise extent of the disease without resorting to unnecessary bone dissection.

\section{Conclusion}

1) The endoscope is a useful adjuvant to the operating microscope in identifying cholesteatoma remnant at the time of primary surgery and ensures better disease clearance.

2) The endoscope is helpful in decision making for limiting dissection while doing cholesteatoma surgeries by the microscope thereby avoiding a large size cavity. 


\section{References}

1. Bauer M (2000) Ossiculoplasty: autogenous bone grafts, 34 years experience. Clin Otolaryngol Allied Sci 25: 257-263. [Crossref]

2. Tarabichi M (2004) Endoscopic management of limited attic cholesteatoma. Laryngoscope 114: 1157-1162. [Crossref]

3. Yadav SP, Aggarwal N, Julaha M, Goel A (2009) Endoscope-assisted myringoplasty. Singapore Med J 50: 510-512. [Crossref]

4. Tarabichi M1 (2000) Endoscopic management of cholesteatoma: long-term results. Otolaryngol Head Neck Surg 122: 874-881. [Crossref]

5. Thomassin JM, Korchia D, Doris JM (1993) Endoscopic-guided otosurgery in the prevention of residual cholesteatomas. Laryngoscope 103: 939-943. [Crossref]

6. Bottrill ID, Poe DS (1995) Endoscope-assisted ear surgery. Am J Otol 16: 158-163. [Crossref]

7. Tarabichi M (1997) Endoscopic management of acquired cholesteatoma. Am J Otol 18: 544-549. [Crossref]

8. Marchioni D, Villari D, Alicandri-Ciufelli M, Piccinini A, Presutti L (2011) Endoscopic open technique in patients with middle ear cholesteatoma. Eur Arch Otorhinolaryngol 268: 1557-1563. [Crossref]

9. Marchioni D, Alicandri-Ciufelli M, Gioacchini FM, Bonali M, Presutti L (2013) Transcanal endoscopic treatment of benign middle ear neoplasms. Eur Arch Otorhinolaryngol 270: 2997-3004. [Crossref]

10. Marchioni D, Alicandri-Ciufelli M, Piccinini A, Genovese E, Presutti L (2010) Inferior retrotympanum revisited: an endoscopic anatomic study. Laryngoscope 120: 18801886. [Crossref]

11. Shelton C, Sheehy JL (1990) Tympanoplasty: review of 400 staged cases. Laryngoscope 100: 679-681. [Crossref]
12. Dodson EE, Hobgood TC (1988) Residual and recurrent cholesteatoma in mastoidectomy operations. Am J Otol 5: 277-282.

13. Biswas D, Mandal K, Roychoudhury A, Roychowdhury R (2014) Otoendoscopy- A new horizon in Ear Sugery. State J Otolaryngol: 4-7.

14. Magnan J, Chays A, Lepetre C, Pencroffi E, Locatelli P (1994) Surgical perspectives of endoscopy of the cerebellopontine angle. Am J Otol 15: 366-370. [Crossref]

15. Pratt LL (1983) Complications associated with the surgical treatment of cholesteatoma Laryngoscope 93: 289-291. [Crossref]

16. Yung MM (1994) The use of rigid endoscopes in cholesteatoma surgery. J Laryngol Otol 108: 307-309. [Crossref]

17. Mohamed MK, Badr-El- Dine M (2009) Surgery of Sinus Tympani Cholesteatoma: Endoscopic Necessity. Int Adv Otol 5: 158-165.

18. Badr-El- Dine M, Shewel YG, Ibrahim AA, Khalifa M (2013) Endoscope-assisted surgery (a major adjunct in cholesteatoma surgery). Egypt J Otolaryngol 29: 66-70.

19. Ayache S, Tramier B, Strunski V (2008) Otoendoscopy in cholesteatoma surgery of the middle ear: what benefits can be expected? Otol Neurotol 29: 1085-1090. [Crossref]

20. Migirov L, Shapira Y, Horowitz Z, Wolf M (2011) Exclusive endoscopic ear surgery for acquired cholesteatoma: preliminary results. Otol Neurotol 32: 433-436. [Crossref]

21. Badr-El- Dine M, El-Garem HF, El-Ashram Y, Talaat AM, Magnan J (2002) Endoscope Assisted Minimal Invasive Microvascular Decompression of Hemifacial spasm. Abstracts of the 9th International Facial Nerve Symposium. Otol Neurotol Suppl 23. $68-72$

22. McKennan KX (1993) Endoscopic 'second look' mastoidoscopy to rule out residua epitympanic/mastoid cholesteatoma. Laryngoscope 103: 810-814. [Crossref]

23. Youssef TF, Poe DS (1997) Endoscope-assisted second-stage tympanomastoidectomy Laryngoscope 107: 1341-1344. [Crossref]

Copyright: (C2017 Verma B. This is an open-access article distributed under the terms of the Creative Commons Attribution License, which permits unrestricted use, distribution, and reproduction in any medium, provided the original author and source are credited. 